\title{
Can Lightning Produce Significant Levels of Mass-Independent Oxygen Isotopic Fractionation in Nebular Dust?
}

\author{
Joseph A. Nuth III $^{1}$ John A. Paquette ${ }^{2,3}$ and Adam Farquhar ${ }^{2,4}$. \\ 'Solar System Exploration Division, Code 690, NASA's Goddard Space Flight Center, Greenbelt MD 20771. \\ ${ }^{2}$ Astrochemistry Laboratory, Code 691, NASA’s Goddard Space Flight Center, Greenbelt MD 20771 \\ ${ }^{3}$ NASA Postdoctoral Fellow ${ }^{4}$ USRP Student Intern.
}

\begin{abstract}
Based on recent evidence that oxide grains condensed from a plasma will contain oxygen that is massindependently fractionated compared to the initial composition of the vapor, we present a first attempt to evaluate the potential magnitude of this effect on dust in the primitive solar nebula. This assessment relies on previous studies of nebular lightning to provide reasonable ranges of physical parameters to form a very simple model to evaluate the plausibility that lightning could affect a significant fraction of nebular dust and that such effects could cause a significant change in the oxygen isotopic composition of solids in the solar nebula over time. If only a small fraction of the accretion energy is dissipated as lightning over the volume of the inner solar nebula, then a large fraction of nebular dust will be exposed to lightning. If the temperature of such bolts is a few percent of the temperatures measured in terrestrial discharges, then dust will vaporize and recondense in an ionized environment. Finally, if only a small average decrease is assumed in the ${ }^{16} \mathrm{O}$ content of freshly condensed dust, then over the last 5 million years of nebular accretion the average $\Delta^{17} \mathrm{O}$ of the dust could increase by more than 30 per mil. We conclude that it is possible that the measured "slope 1" oxygen isotope line measured in meteorites and their components represents a time-evolution sequence of nebular dust over the last several million years of nebular evolution where ${ }^{16} \mathrm{O}$-rich materials formed first, then escaped further processing as the average isotopic composition of the dust gradually became increasingly depleted in ${ }^{16} \mathrm{O}$.
\end{abstract}




\section{Introduction}

The remarkable discovery that oxygen isotopes in primitive meteorites had $\delta^{17} \mathrm{O}$ vs. $\delta^{18} \mathrm{O}$ that plotted along a line of slope 1 rather than along the typical slope 0.52 equilibrium fractionation line (see Figure 1) was published almost 40 years ago (Clayton, Grossman and Mayeda, 1973). However, a satisfactory, quantitative explanation for this observation has yet to be found, though many different explanations have been proposed. The original problem was not only to find a process to distribute oxygen isotopes along a slope 1 line, but also to find a mechanism that simultaneously increased the ${ }^{16} \mathrm{O}$ content of the dust. This implicitly assumed that the oxygen isotopic composition of the Earth, Mars and most meteorites was close to the composition of the dust in the protosolar nebula.

The first of these explanations proposed after the discovery of this phenomenon was that the observed line represented the result of mixing molecular cloud dust with a nucleosynthetic component, rich in ${ }^{16} \mathrm{O}$, possibly resulting from a nearby supernova explosion. This would require that a large fraction of the solar nebula ( $\sim 5 \%$ by mass) originated in that supernova (Cameron and Truran, 1977). As an alternative to the supernova hypothesis, Donald Clayton (1988) suggested that galactic chemical evolution would gradually change the oxygen isotopic composition of the interstellar grain population by steadily producing ${ }^{16} \mathrm{O}$ in supernovae, then producing the heavier oxygen isotopes as secondary products in lower mass stars. Thus presolar materials might start with oxygen isotopes already distributed along the slope 1 line.

Thiemens and collaborators proposed a chemical mechanism that relied on the availability of additional active rotational and vibrational states in otherwise-symmetric molecules, such as $\mathrm{CO}_{2}, \mathrm{O}_{3}$ or $\mathrm{SiO}_{2}$, containing two different oxygen isotopes (Thiemens and Jackson, 1987; Thiemens, 1996). He demonstrated in the laboratory that a slope 1 distribution of oxygen isotopes could be produced by an electrical discharge (Heidenreich and Thiemens, 1985). He also proposed another process, suggesting that differential photochemical dissociation could fractionate oxygen isotopes (Thiemens and Heidenreich, 1983). Mauersberger and colleagues (Mauersberger et al., 1999) discovered this effect in the terrestrial stratosphere and demonstrated a kinetic route to the atmospheric results in the laboratory (Krankowsky and Mauersberger, 1996). A photochemical approach to understanding the slope 1 distribution in solar system solids has been pursued by several groups (Clayton, 2002; Lyons and Young, 
2005; Yurimoto and Kuramoto, 2004). Though none of the current models have yet reached a stage where it can produce quantitative results to explain observations of slope 1 solids using processes relevant to the solar nebula, all can produce a slope 1 oxygen isotopic distribution in water produced following photochemical dissociation of CO. It is simply assumed that this water then reacts with dust to yield the isotopic results observed in meteorites.

Marcus and colleagues (Hathorn and Marcus, 1999; 2000; Gao and Marcus, 2002) proposed a quantum chemical explanation for the slope 1 isotopic distribution observed following the formation of ozone. Marcus (2004) also proposed a quantum chemical model to explain the oxygen isotopic distribution of solar system solids as they condensed from the vapor in a series of surface mediated reactions. In this second model the oxides incorporated into the grain became richer in ${ }^{16} \mathrm{O}$ relative to the gas phase. If the oxygen isotopic composition of nebular solids is represented by that of Standard Mean Ocean Water (SMOW), then this model might produce the ${ }^{16} \mathrm{O}$-enriched Calcium Aluminum Inclusions (CAIs) and chondrules distributed along the observed slope 1 line.

However, the oxygen isotopic composition of the sun, as measured by the Genesis Mission (McKeegan, et al., 2011) is considerably enriched in ${ }^{16} \mathrm{O}$ relative to the composition of terrestrial oxygen as exemplified in SMOW. The general consensus is that since the sun represents the largest oxygen reservoir in the solar system, then it must also represent the average isotopic composition of the dust and gas in the solar nebula. Krot et al. (2010) suggested that dust had an isotopic composition close to that of most meteorites and the terrestrial planets and that the gas had an isotopic composition slightly more ${ }^{16} \mathrm{O}$-rich than the sun. Based on solar abundances, $26 \%$ of solar system oxygen was in dust and $74 \%$ in gas. CAIs, which are closer to the sun in oxygen isotopic composition, would therefore represent equilibration between gas and dust, whereas the dust that made up most of the solar system rocks we have measured did not equilibrate with gas. This difference between primordial gas and dust might have arisen by galactic chemical evolution or pollution of the protosolar molecular cloud by massive star ejecta.

If such a primordial difference did not exist, then one solution might be producing ${ }^{16} \mathrm{O}$ depleted solids to match the observed composition of solar system bodies under the assumption that the dust and gas in the nebula had an initial average isotopic composition equal to (or at least very close to) that of 
the sun but that the initial dust and gas reservoirs in the molecular cloud evolved very distinct isotopic compositions with the dust composition rich in ${ }^{16} \mathrm{O}$ and the gas depleted. Dominguez (2010) has suggested that chemical reactions on cold surfaces could produce "molecular cloud $\mathrm{H}_{2} \mathrm{O}$ and possibly other IS solids inherited from the molecular cloud (that) were depleted in ${ }^{16} \mathrm{O}$ compared to the bulk gasphase $\mathrm{O}$ present, thus providing distinct ${ }^{16} \mathrm{O}$ reservoirs at the earliest stages of planetary formation." The predictions of this model could be difficult to distinguish from those of the photochemical-selfshielding model of Yurimoto and Kuramoto (2004) discussed below.

We had previously predicted (see Wiens et al., 1999; Nuth et al., 1998) that the oxygen isotopic composition of the sun is more ${ }^{16} \mathrm{O}$ rich than $(-40,-40)$ per mil relative to SMOW and had suggested that the oxygen isotopic composition of solar system solids found in meteorites and even in planets must have been the result of continuous processing of dust in the solar nebula over time (Nuth et al, 1998) as illustrated in Figure 1. Based on experiments (Kimura, et al., 2007) that produced non-massdependently fractionated solid oxides when silicon and iron condensed from oxidizing plasma, we have argued (Nuth, 2009) that time-dependent processing of nebular dust via nebular lightning could explain the observed oxygen isotopic distribution in the solar system. Note that unlike Photochemical Self Shielding, this model does not produce a slope 1 line, ab initio, but rather gradually increases the $\Delta^{17} \mathrm{O}$ of the dust over time. Surviving remnants such as some CAIs can be used to trace out the isotopic path of the dust while the bulk composition (on average) becomes increasingly depleted in ${ }^{16} \mathrm{O}$. This will be discussed in more detail below.

The conditions necessary for nebular lightning have been previously discussed (Gibbard, et al., 1997; Love, et al., 1995; Desch and Cuzzi, 2000). In what follows we will present a rough model of the time dependent evolution of oxygen isotopes in nebular dust based on processing by lightning; however, we note that there is a wide range in the possible values of many of the required input parameters for this model. In many instances, the exact value of a single parameter could change significantly but its effect could be offset by a change in one or more of the other inputs to the model. We expect that future work will narrow the range in the uncertainties in the model's parameters and that such constraints will lead to a deeper and more detailed understanding of the dynamics and chemistry of protostellar systems. 
Below we will briefly outline the main assumptions of the model and present the results of these calculations for what we believe to be a plausible set of input parameters. We will then briefly summarize the main results of the model and discuss the range of likely uncertainties in the most important input parameters as well as the potential factors which could offset the effects of changes in these variables. Finally we will compare and contrast the results of lightning induced oxygen isotopic fractionation with the leading model for producing the observed slope 1 oxygen isotope line, photochemical self shielding.

\section{A Model of Nebular Lightning and Oxygen Isotopic Fractionation}

We will assume that materials that may have been processed through the initial collapse phase of the solar nebula were nearly all incorporated into the sun and that only the tail end of the accretion process left material that had any chance of being incorporated into planetesimals. This implies that we should start calculations of dust processing roughly at "time zero," the time when CAIs formed with a full complement of ${ }^{26} \mathrm{Al}$. While we assume that the dust (and gas) at this time both had solar oxygen isotopic composition (on average), it is also possible that previous, and much more active processing of the dust by lightning in earlier stages of accretion could already have significantly fractionated some quantity of nebular dust with respect to the gas. However, since we are trying to evaluate the potential fractionation of the dust left in the minimum mass disk at the end of the accretion process, we will adopt the worst case assumption (for our model) that no ${ }^{16} \mathrm{O}$-depleted dust survives in the disk from previous processing that may have occurred before this final stage of nebular evolution.

\section{The frequency of nebular lightning}

We assume for simplicity that the energy dissipated in the nebula due to lightning is a fixed fraction of the instantaneous accretion energy, and that the accretion rate of the nebula exponentially decreases with time as the sun accumulates the last few percent of its mass. For the model presented here we will assume that $5 \%$ of the accretion energy is dissipated as lightning. This assumption is consistent with previous estimates (Gibbard, et al., 1997; Love, et al., 1995; Desch and Cuzzi, 2000), but could be off in either direction. In addition, the fraction of accretion energy dissipated as lightning might not 
actually be a constant. Both tribo-electric charging and turbulent charge separation depend to some degree on accretion energy, so that lightning may have a much stronger dependence on accretion energy than we have specified in this model. Were this true, then the isotopic fractionation of the solids would occur at an even faster pace earlier in our model but would taper off much faster at the end of the simulation. In addition, we have not taken into account the possible effects of magnetic reconnection of twisted solar field lines in producing additional electrical discharges in the nebula that could provide another source of plasma dust processing (Hawley and Stone, 1998). Discharges due to magnetic reconnection events might not have any dependence on accretion rate but could instead provide a relatively steady background level of dust processing and evolution. A more quantitative assessment of the rate of electrical discharges in the solar nebula would be extremely useful.

In order to calculate the accretion energy available to power nebular lightning we need to define the mass of this final nebular phase and the timescale over which mass falls through the nebular disk and (mostly) into the sun. For this model we assume that 0.039 solar masses ( 3 times the minimum mass solar nebula) falls into and through this disk at an exponentially decreasing rate over a 5 million year period leaving a final mass of at least 0.013 solar masses from which to form the observed bodies in the solar system. Thus the rate of infall is given by

$$
\frac{d M}{d t}=6 \times 10^{-8} \exp \left(\frac{-t}{6.5 \times 10^{5}}\right)
$$

in solar masses per year. Time, $t$, is measured in years. The energy per unit time due to infall as a function of time is

$$
\frac{d U}{d t}=7.22 \times 10^{33} \exp \left(\frac{-t}{6.5 \times 10^{5}}\right)
$$

in ergs per second.

Both the exponential decrease in energy and the timescale of the decrease in the model were chosen to match the available data (Bell, et al., 1997). However, it is possible that the nebula could have settled into a reasonably steady state of mass influx after an initial rapid rate of accretion rather than the 
exponential decrease that we assume in this model. Indeed, other prescriptions for the accretion rate towards the end of the infall are available (Hersant, et al., 2001; Hartmann, et al., 1998). However, given the range of uncertainties in all of our input parameters, the most obvious effect of using these alternative accretion rate prescriptions is a change in the time dependence of nebular lightning. A more detailed model of stellar accretion that spans the transition from star formation, through the disk phase to disk dissipation would aid in determining the time available for dust processing and, if such a model were validated, would help to constrain the other input parameters.

In order to keep the model as simple as possible, we assume that the mass falling through the minimum mass nebula does not change the composition of the dust in the disk either by adding unprocessed materials to the outer regions of our model volume or by concentrating processed materials in the inner region of the disk. We also assume that nebular material is extremely $(25 \%)$ efficient in forming planetesimals at this stage of nebular evolution and that the rest of the material present in the disk volume that does not go into planetesimals either falls into the sun or is dispersed in the bi-polar outflows or T-Tauri wind. In order to calculate the fraction of the nebula affected by each lightning bolt we need to define the volume of the nebula from which the planetesimals will accrete. For this model we will assume that all processing occurs in an inner disk 5 A.U. in radius and 1 A.U. thick from which the majority of rocky planetesimals will eventually accrete. This gives a volume for the inner nebula in cubic meters of

$V_{N}=\pi \times\left(5 \times 1.5 \times 10^{11}\right)^{2} \times 1.5 \times 10^{11}=2.7 \times 10^{35}$

We further assume that the dust and gas in this volume is uniformly mixed and distributed; e.g., there has been no dust settling to the mid-plane. Note that a model using a flared disk 1 A.U. thick at 5 A.U. or that assumed considerable settling of dust to the midplane where most of the lightning would occur would be much more efficient at processing the dust than we have assumed here.

In order to calculate the frequency of lightning bolts as a function of time we will define a standard length and channel diameter for each stroke and thereby calculate the energy for each individual bolt. For the physical characteristics of the lightning bolt, we have relied on the work of Desch and Cuzzi (2000) for the typical input parameters used in this model. The minimum length of a typical lightning 
bolt in the solar nebula was assumed to be $100 \mathrm{~km}$, and the radius of the lightning channel was assumed to be 10 times the electron mean free path length $(0.7 \mathrm{~m})$ at a typical nebular pressure $\left(10^{-5}-10^{-6}\right.$ bar). Thus the volume of a lightning bolt in cubic meters is

$$
V_{L}=\pi \times 7^{2} \times 100,000=1.54 \times 10^{7}
$$

Both longer and shorter strokes are certainly possible. However, since we calculate the lightning frequency based on available energy, changing the length of the bolt will change the energy dissipated and thus the overall number of discharges per second at any given time, but will not change the total volume processed through lightning bolts. Similarly, changing the width of the discharge channel changes the energy expended in a single stroke, but also changes the volume of material processed per bolt. Therefore, due to the way our model is constructed, changes to the physical dimensions of the standard lightning bolt itself will not change our results. We have used a mean value of 10 times the electron mean free path for the width of the discharge channel in our calculations whereas terrestrial lightning channel widths could be up to 1000 times the electron mean free path. Desch and Cuzzi (2000) estimate that values up to 100 times the electron mean free path are quite plausible for the solar nebula. Given all of the assumptions stated above, the number of lightning bolts per second over the entire nebular volume considered in this model is

$$
\frac{d N}{d t}=1.1 \times 10^{16} \exp \left(\frac{-t}{6.5 \times 10^{5}}\right)
$$

and the cumulative number of bolts between time zero and time $t$ is

$$
N(t)=2.3 \times 10^{29^{!}}\left[-\exp \left(\frac{-t}{6.5 \times 10^{5}}\right)\right]
$$

The frequency of lightning bolts over the entire nebular volume considered in this model is shown in Figure 2 . 


\section{Isotopic fractionation of nebular dust}

We first assume that an individual lightning bolt vaporizes all of the dust within its ionization channel and brings the gas and dust in the channel to a temperature of at least $2000 \mathrm{~K}$. We note that the temperature of a terrestrial lightning bolt is about 30,000K (Lide, 1996). While we have assumed that all of the dust within the discharge channel is vaporized, it is likely that considerable quantities of dust outside the channel are also vaporized. Ionized metals, gases and electrons will diffuse from the lightning channel into these surrounding regions (or be formed outside the channel itself due to intense radiation from the lightning stroke or by shocks) where more vaporized dust will also nucleate in a plasma environment, albeit at a lower ionization state than in the channel itself. It is therefore probable that an estimate of the volume of dust affected by a single lightning bolt in the current model would be a lower limit if we simply used the volume of the lightning bolt itself. Preliminary calculations indicate that complete dust vaporization can occur out to at least 5 times the radius of the ionization channel, assuming a channel temperature of only $2000 \mathrm{~K}$, though the ionization state of the gas and the induced fractionation throughout such a volume is highly uncertain. It is therefore possible, though not necessarily probable, that a single lightning bolt could process a dust volume that is much greater than the volume of a single lightning bolt for the same energy expenditure, especially if the temperature of the ionization channel is significantly higher than we have assumed. To account for this, a lightning bolt is assumed to process dust in a radius three times greater than the radius of the lightning stroke itself. Of course, for our model, if the temperature were higher, then either the energy per bolt would increase and the lightning frequency decrease, or the size of the bolt would decrease so that the energy of each bolt remained the same in order to maintain a constant fraction of the accretion energy.

A basic assumption of this model is that the oxygen content in dust that recondenses from a plasma is non-mass dependently fractionated to some degree. For this model we will assume, based on the results of the experiments cited earlier (Kimura, et al., 2007), that the oxygen isotopic composition of recondensed dust is depleted in ${ }^{16} \mathrm{O}$ by 0.5 per mil compared to the starting material and that the excess ${ }^{16} \mathrm{O}$ is incorporated into gas phase molecules such as $\mathrm{CO}, \mathrm{CO}_{2}$ and $\mathrm{H}_{2} \mathrm{O}$. We can then either assume that dust and gas in a specific volume element remains locally distinct or we can assume that fractionated dust from each individual lightning stroke is "instantaneously" mixed throughout the nebula to maintain an average oxygen isotopic composition for nebular dust at any given time. In 
reality, as we assume that lightning occurs randomly throughout the entire volume of the nebula based on the available energy and because the initial frequency of lightning strokes throughout the nebula is extremely high (see Figure 2), the required mixing time to distribute the products of any given lightning bolt over the model disk to create an average oxygen isotopic composition of the dust is much shorter than the mixing time scale for the nebula itself. Boss (2004) has previously demonstrated that even very large-scale heterogeneity in the solar nebula is damped out within a few thousand years, and therefore the mixing that we envision in this model should be quite rapid. Note that while we expect that the large-scale heterogeneity of dust in the nebula will be eliminated by mixing, small scale heterogeneity could persist for much longer times (e.g., see Richter, 1984).

If the change in oxygen isotopic fractionation per bolt is $\Delta f_{L}$ then the cumulative change in oxygen isotopic fractionation over the whole nebula is given by

$$
\Delta f_{N}=9 \frac{V_{L}}{V_{N}} \Delta f_{L} N(t)
$$

Here $V_{L}$ and $V_{N}$ are the volumes of a single lightning bolt and of the inner nebula, respectively. We assume a conservative value of $\Delta f_{L}$ of only $0.5 \%$ in this work, less than seen by Kimura, et al. (2007). While these experiments used a combination of $\mathrm{SiH}_{4}, \mathrm{Fe}(\mathrm{CO})_{5}, \mathrm{O}_{2}$ and $\mathrm{H}_{2}$ rather than silicate dust as the input to the electrical discharge, the discharge acted to dissociate the gas to form $\mathrm{Si}, \mathrm{Fe}, \mathrm{H}$ and $\mathrm{O}$ atoms just as would be formed from the vaporization of silicate dust in a hydrogen rich nebula. In addition, while these experiments showed mass independent fractionation effects in excess of 4 per mil in $\Delta^{17} \mathrm{O}$, such effects were accompanied by considerable levels of mass dependent fractionation as well, and the mass dependent fractionation appeared to vary systematically with the composition of the condensate. We believe that several species likely formed in the plasma could be responsible for the mass independent effects observed in the experiments including: $\mathrm{O}_{3}, \mathrm{OH}$ and $\mathrm{CO}_{2}$. However, both for simplicity, and because we do not yet have a robust chemical model to account for the composition of the oxides that condensed in the plasma, we do not include mass dependent fractionation effects in these calculations.

Figure 3 shows the change in oxygen isotopic composition of nebular dust as a function of time given the assumptions listed above and starting at an arbitrary composition. The figure shows that the 
oxygen isotopic composition of the dust can evolve from an arbitrary value to a $\delta^{17} \mathrm{O}, \delta^{18} \mathrm{O}$ as much as 50 per mil higher within much less than a million years after time zero, and rises much more slowly thereafter. This slowdown is due to the significant decrease in energy available for lightning to process nebular dust as the nebular accretion rate drops. If the average planetesimal that went into the primitive Earth, Mars or meteorite parent body does not form until at least a few hundred thousand years after time zero, then Figure 3 demonstrates that a lightning driven model of dust processing is capable of producing the materials from which these bodies accreted starting from approximately solar composition $(-40,-40)$ after being processed through myriad lightning strokes. In this model the bulk of nebular dust would evolve to near the terrestrial oxygen isotopic composition on a very rapid timescale. The CAIs and chondrules found along the slope 1 line would represent residual small-scale remnants of processed dust from earlier times incorporated into later-forming parent bodies.

The CAIs and some chondrules could be rare intermediates that may have been preserved by being transported outward after their formation from nebular dust (Ciesla, 2010) and then fallen back much later to be incorporated into growing planetesimals after the rate of nebular accretion had dropped significantly and lightning driven oxygen isotopic evolution of the dust had slowed. The rate at which accretion tails off is a very important, but poorly constrained part of this model. Furthermore, Ciesla $(2007 ; 2010)$ has demonstrated that the outward flow of materials (such as CAIs) from the inner nebula is strongest while the accretion rate is highest. The flow of processed materials, both CAIs and chondrules outward or isotopically fractionated dust and larger grains back into the sun will determine the final composition of the meteorites and planetesimals. As an unlikely alternative, it is also possible that the CAIs and chondrules along the CAI line represent small-scale heterogeneities that somehow survived further nebular processing in place (Paquette and Nuth, 2012).

We can also compute the change in oxygen isotopic composition for specific nebular volumes by assuming that no mixing occurs and that the total degree of isotopic fractionation with respect to the starting composition is determined simply by the number of lightning strokes experienced by that volume on a totally stochastic basis. Figure 4 shows the mean number of times that dust in 10,000 distinct, closed volumes were struck by lightning as a function of time for the assumptions as stated above. For the purpose of Figure 4 it is assumed that no mixing occurs between volumes. Also shown are the values one standard deviation from the mean and the minimum and maximum number of bolts 
that struck any reference volume. For each volume at each time step the probability of a lightning strike was computed based on the frequency of lightning bolts at that time and the volume of the model nebula thus affected. A random number between zero and one was generated and then compared to the probability of a lightning strike. The model tracked the total number of strikes in each volume as a function of time for the first five million years after time zero.

Figure 5 shows the distribution of the number of strikes per unit volume at several selected times ranging from 10 years after time zero up to 5 million years after the start of the run. As can be seen, even as late as 10,000 years after the start of the calculation there are some volumes that have not been processed at all. At 100,000 years all volumes have experienced at least several lightning strikes while the average volume has been struck 20 times. At increasing times the distribution of strikes becomes more symmetric and the width of the distribution increases slightly. By the end of the calculation there is nearly a $50 \%$ difference between the number of lightning strokes affecting volumes with minimum and maximum numbers of hits. If the degree of isotopic fractionation is directly proportional to the number of times a given dust grain vaporizes and recondenses in plasma, then this would indicate a significant spread in the oxygen isotopic composition of the average grains from volume to volume. While we believe that significant mixing is inevitable in a turbulent, evolving nebula, these calculations indicate that there could be large deviations from the average isotopic composition of the dust in isolated volumes of the nebula. Of course, as the dust aggregates into planetesimals much of this divergence will revert to the mean (Nuth and Hill, 2004).

\section{Discussion}

There are many uncertainties in the input parameters for this model, and in many (but not all) cases we have tried to err by using the more unfavorable values for such parameters. However, many of these uncertainties should be amenable to further refinement. As an example, experimental studies of non-mass dependent fractionation as a function of vapor composition and ionization state would be extremely useful in quantifying the conditions and degree of isotopic fractionation for specific refractory vapors as a function of distance from the lightning discharge channel. Chemical models of the fractionation process itself on a molecular level, physical models of lightning generation in a turbulent nebula, dynamic models of the accretion rate during the final disk phase from the time of CAI 
and chondrule formation through to the formation of large planetesimals would all be useful in understanding the timescale and efficiency required to reproduce the observed isotopic composition of solar system objects. Observations of the accretion rate during the final disk forming stage of protostellar evolution or measurements of the frequency of nebular lightning as a function of accretion energy in modern protostars would greatly constrain the problem. We readily acknowledge that there are many parameters of this model that need refinement. Nevertheless, the ballpark estimates used to construct the present model suggest that a time-dependent understanding of the oxygen isotopic composition of solids in the solar nebula can be developed based on lightning processing of dust. The model also suggests that the vast majority of the isotopic changes in the dust occur very rapidly (e.g., within the first half-million years of nebular evolution) and prior to the accretion of the large numbers of planetesimals that eventually became the Earth, Moon or Mars, or that represent the population of asteroids that are contained within our museums as meteorites.

\section{The survival of presolar grains}

One interesting corollary of the stochastic calculations presented in Figures 4 and 5 might be the conclusion that presolar grains could not survive in the solar nebula, yet they do exist. We believe that the answer to this problem lies in our assumption that no mixing occurs as fresh material falls through the minimum mass nebula and into the sun. While it is convenient from a modeling perspective to ignore such mixing, it goes against our physical intuition. If fresh "solar composition" dust falls onto the outer portions of the disk then it will dilute the degree of isotopic processing that evolved from time zero. In a similar vein, if processed dust falls towards the sun, it could enrich the dust of the inner nebula in ${ }^{16} \mathrm{O}$-depleted dust. The overall effect would be to produce a gradient in the oxygen isotopic composition of the dust such that the innermost nebula contained more ${ }^{17} \mathrm{O}$ and ${ }^{18} \mathrm{O}$ that gradually blended into the more ${ }^{16} \mathrm{O}$-rich dust of the outer nebula. While this is the opposite of what models that create separate oxygen isotopic reservoirs in the parent molecular cloud would predict, it could be consistent with self-shielding models driven by solar radiation (see below). Could the vigorous nebular mixing suggested by Boss (2004) or the outward flow of nebular gas and solids suggested by Ciesla $(2007 ; 2010)$ erase such a gradient? 
In order to compute the effect of mixing fresh materials into the nebula that might contain presolar grains, we have done something that is impossible in the real world: We have run time in reverse. In particular, we have used our stochastic approach to calculate the probability of survival of a volume of material that drops into the nebula at a point in time, starting at 5 million years after time zero and progressing back until it is struck by lightning. Since the rate of lightning strikes in our model increases exponentially as we go back in nebular time, all such packets are eventually vaporized. However, Figure 6 demonstrates that it is possible for a packet of material to fall into the solar nebula just two million years after time zero and still survive unscathed under the assumptions of the current model. Obviously, material added later has a much better chance for survival due to the decreased rate of lightning strikes, though the amount of such additional material also decreases drastically as a function of time due to the decreased rate of accretion.

Future models need to account for the retention of fresh, unprocessed nebular materials within the final minimum mass solar nebula, and might possibly need to start calculations some time before time zero in order to retain some of the highly processed grains from the era of even higher frequency lightning activity that could have significantly "pre-processed" a small fraction of nebular dust that did not fall into the sun.

\section{Comparison of the effects of lightning with those of photochemical self-shielding}

While we have constructed this model of the oxygen isotopic evolution of nebular dust based solely on the processing of such dust via lightning, it is certainly possible that other chemical processes such as photochemical self shielding could play a role in producing dust containing increasingly fractionated oxygen. We have built a model of the simple, lightning-only processing of nebular dust to examine the effects of this process in isolation. We would expect that similar frameworks could be constructed to evaluate the feasibility of any process proposed to change the oxygen isotopic composition of nebular dust. A simple model of one version of the photochemical self-shielding mechanism has been evaluated and found to be generally compatible with observations (Young, 2007). It would be informative to construct a comprehensive model for oxygen isotopic fractionation of nebular dust that accounts for all of the possible processes that have been proposed to explain this observation. 
Photochemical Self-Shielding models come in at least three distinct varieties with different degrees of commonality. All rely on the exhaustion of ${ }^{12} \mathrm{C}^{16} \mathrm{O}$ dissociative photons as the radiation source penetrates through the gas and this fractionation can be calculated precisely for each dissociative band of CO. In the first case (Yurimoto and Kuramoto, 2004), stellar radiation penetrating into the natal cloud from which the solar system collapsed produces a gas enriched with ${ }^{17} \mathrm{O}$ and ${ }^{18} \mathrm{O}$ towards the interior of the cloud that could be transferred first into $\mathrm{H}_{2} \mathrm{O}$, then into the silicates during subsequent nebular grain processing events such as shocks. Such a model produces regions within the cloud where, on average, the oxygen isotopic composition of water ice would be distinctly ${ }^{16} \mathrm{O}$-poor compared to the oxygen isotopic composition of the gas. In particular the model assumes that most of the ${ }^{16} \mathrm{O}$-depleted oxygen combines with abundant hydrogen to produce water or ice and that the $\mathrm{CO}$ in these regions would be significantly enriched in $\mathrm{C}^{16} \mathrm{O}$. Assuming that the dust does not separate from the gas during collapse, this would represent the starting composition of the nebula.

In the other two versions of the self-shielding model, the source of the $\mathrm{C}^{16} \mathrm{O}$ dissociative photons is the energetic young sun. In one version (Lyons and Young, 2005) the radiation preferentially dissociates $\mathrm{C}^{17} \mathrm{O}$ and $\mathrm{C}^{18} \mathrm{O}$ in the flared nebular disk. The resulting oxygen atoms react with hydrogen to produce water or ice that must then react with dust grains following convection deeper in the nebula towards the midplane. As in the first model the gas is enriched in $\mathrm{C}^{16} \mathrm{O}$ that will remain with the solids barring a mechanism for dust-gas separation. In the second version (Clayton, 2002), solar radiation penetrates directly into the disk near the midplane producing a population of ${ }^{16} \mathrm{O}$-depleted oxygen atoms at some nebular radius (dependent on gas density and dust opacity) that could react directly with under-oxidized dust that might form via the vaporization and recondensation of refractory materials (Nuth, Rietmeijer, and Hill, 2002) in the high temperature regions near the sun. As in the previous version of the process, the gas is enriched in $\mathrm{C}^{16} \mathrm{O}$ that is assumed to be an extremely stable sink for ${ }^{16} \mathrm{O}$. Unfortunately, this is not true for a dusty, hydrogen-rich environment such as the solar nebula. In fact, such environments present ideal conditions for the Fischer-Tropsch reaction that will convert CO into hydrocarbons, releasing the enriched ${ }^{16} \mathrm{O}$ back into the gas phase as water:

$\mathrm{CO}+3 \mathrm{H}_{2}+$ dust $=>\mathrm{CH}_{4}+\mathrm{H}_{2} \mathrm{O}+$ dust 
The Fischer-Tropsch mechanism has long been seen as a potential route to meteoritic organics (see, e.g. Hayatsu and Anders, 1981) and such surface mediated reactions are still under investigation (Llorca and Casanova, 2000; Hill and Nuth, 2003). A series of papers (Nuth et al., 2008a, b) has recently shown that any solid surface can promote Fischer-Tropsch Type (FTT) reactions, even graphite (Nuth, et al., 2010). A more interesting finding is that the FTT reactions appear to leave an organic film on the grain surface that is a more effective catalyst than the original material, thus greatly increasing the efficiency of FTT reactions in converting $\mathrm{CO}$ and $\mathrm{H}_{2}$ into organic materials. If FTT reactions are important in the inner solar nebula then $\mathrm{C}^{16} \mathrm{O}$ is not a stable repository for ${ }^{16} \mathrm{O}$. In fact, FTT processes operating on the residual $\mathrm{CO}$ population will release the oxygen back into the nebula as $\mathrm{H}_{2} \mathrm{O}$, which will mix with the water produced via self-shielding to reconstitute the original oxygen isotopic distribution.

In related work, Ciesla and Sandford (2011) have demonstrated that the thermal and radiation environment in the outer solar system is ideal for the conversion of $\mathrm{CO}$ trapped in ices into complex organic molecules. Such CO-rich ice coatings could form on the edge of the inner nebula and be transported outward for processing or they may have formed in the pre-collapse molecular cloud. The organics (and water) thus formed would eventually filter back into the inner solar nebula where these organic coated grains would be incorporated into growing planetesimals and the water ice would mix with that formed via self-shielding, again counteracting the isotopic fractionation originally achieved. Of course, as these planetesimals evolve due to internal heating or collisional processes, the organics will react with oxidized metals to produce $\mathrm{CO}$ that will be released back into the nebular gas.

From the above we can deduce that there should be a significant carbon cycle operating in the inner nebula. Organic coated grains are either heated directly via solar radiation, lightning or shocks or they are incorporated into thermally evolving planetesimals where they react to generate copious quantities of $\mathrm{CO}$ or $\mathrm{CO}_{2}$ that escapes back to the nebula. Once back in the gas phase these oxides have at least two paths that can reform organics on grain surfaces to start the cycle over again. The $\mathrm{C}^{16} \mathrm{O}$ left intact by the photochemical self shielding mechanism and not processed by either FTT reactions or radiation within icy grain mantles should mix with $\mathrm{CO}$ generated by planetesimal evolution, greatly diminishing the effectiveness of the self shielding process in altering the isotopic composition of nebular dust. In 
addition, the water, $\mathrm{CO}$ and dust will also be vaporized by lightning strikes, shocks or solar flares, further mixing the components of the gas and dust populations.

Lightning-induced oxygen isotopic fractionation enjoys several advantages over photochemical self shielding. First, there is direct experimental evidence that condensation of metal oxide vapors from a plasma results in non-mass dependently fractionated solids that are depleted in ${ }^{16} \mathrm{O}$ compared to the oxygen in the initial dust. No experimental evidence yet exists that mass independently fractionated refractory oxide grains can be produced as the result of self shielding, though it is well known that self shielding can produce a slope 1 oxygen isotopic fractionation line in products formed after the differential photodissociation of CO (Shi, et al., 2012). Second, lightning produces fractionated grains directly; no transport or secondary reactions are required. Most self shielding models produce gas enriched in ${ }^{17} \mathrm{O}$ or ${ }^{18} \mathrm{O}$ atoms that must then either react with dust directly or must react with hydrogen to form water, be transported to a location where the water can react with dust, then react to produce grains deficient in ${ }^{16} \mathrm{O}$. The great disadvantage of the lightning model is that there is currently no chemical mechanism to explain why refractory oxides condensed from plasma contain oxygen that is isotopically fractionated to the degree observed. The self shielding model has the advantage in this regard as there is, at least on paper, a simple chemical mechanism that adequately explains the production of a gas phase enriched in ${ }^{17} \mathrm{O}$ or ${ }^{18} \mathrm{O}$ atoms together with a proposed chemical route to get these enriched atoms into the dust (see Lyons and Young, 2005; Young, 2007). While we might question the assumptions of this mechanism or its applicability to the conditions in the solar nebula, we must also acknowledge that no chemical model yet exists to explain even the fractionation observed in the laboratory for plasma produced refractory oxides.

In contrast to traditional wisdom we believe that the production of a slope 1 oxygen isotopic fractionation line by the self-shielding mechanism is actually a disadvantage of the model and is inconsistent with observations when compared to the time-dependent lightning-induced fractionation model. The time-dependent model predicts the evolution of the oxygen isotopic composition of the dust from one that is solar to a ${ }^{16} \mathrm{O}$-depleted state as dust is processed in an exponentially decreasing energetic environment. Such an environment should reprocess older grains more rapidly than ones produced later. Thus the grains closest to solar represent fortunate (relatively rare) survivors of this process formed early in solar system history while the bulk of nebular dust evolved to a more ${ }^{16} \mathrm{O}$ - 
depleted state. In addition, there is a logical maximum deviation from the solar composition that will be produced by a time-dependent model due to an exponentially diminishing rate of lightning.

In contrast, there is no time dependence in the current self-shielding models, and all of these models distribute the oxygen isotopic composition of the oxygen reservoir uniformly along the canonical slope 1 line. In such models there is no reason why the oldest (first formed) solar system materials would show a composition closer to solar than later-formed planetesimals. If self-shielding raised the average composition of dust in the nebula to near SMOW, then one should expect to find both CAIs and chondrules distributed both above and below the intersection of the slope 1 and terrestrial fractionation lines. Indeed, one might expect to find planetesimals distributed along this line as well. While one might invoke mixing to argue that the isotopic compositions of such planetesimals would revert to the mean as they accreted (e.g., Nuth and Hill, 2004), the observed diversity in the isotopic composition of meteorite groups would appear to limit the effectiveness of this process, concentrating the dust composition of planets and planetesimals near SMOW and well away from that of the sun. However, if these same meteorite groups formed at different times from the average dust present in the nebula at those specific moments, then one would naturally predict that each planetesimal would have an isotopic composition representative of the time (and possibly the place) where it formed. The difference between the isotopic compositions of these bodies and that of the sun would also be explained by the timing of the specific accretion process.

\section{Chronology, a Supernova Trigger and Short-lived Radio Isotopes}

The exact timing of events in and materials from the early solar nebula is still a matter of debate (Young, et al., 2005). There are several observations which are difficult to reconcile with any of the models of oxygen isotopic fractionation. Some CAIs and one chondrule are ${ }^{16} \mathrm{O}$-enriched relative to the sun (Gounelle M., et al., 2009; Kobayashi S., et al., 2003). Could this be evidence for isotopic fractionation of solar composition dust by the mechanism proposed by Marcus (2004)? Although we have argued for a monotonic increase in oxygen isotopic composition with time (Nuth et al., 1998 and

references therein), evidence against such an evolutionary sequence exists (see below) and the situation is definitely unclear. For example, although CAIs are ${ }^{16} \mathrm{O}$-enriched relative to other solar system solids, differentiated meteorites (which may have accreted prior to chondrule formation) are generally 
less ${ }^{16} \mathrm{O}$-enriched compared to chondrules. One of the oldest chondrules in ordinary chondrites is ${ }^{16} \mathrm{O}$ depleted relative to younger chondrules in carbonaceous chondrites (e.g., in CRs; chondrule ages are based on ${ }^{26} \mathrm{Al}^{26}{ }^{26 g}$ chronology; see Kobayashi et al., 2003). In addition, it appears that ${ }^{16} \mathrm{O}$-rich and ${ }^{16} \mathrm{O}$-poor reservoirs coexisted during the epoch of CAI formation (Simon et al., 2011; Itoh and Yurimoto, 2003). However, it is possible that the oldest solids in the solar system may have formed before ${ }^{26} \mathrm{Al}$ was fully incorporated into the solar nebula. These might also represent examples of materials condensed from volumes at the extremes of stochastic dust processing where very large differences in oxygen isotopes exist contemporaneously - at least until global mixing more thoroughly homogenizes dust on larger spatial scales throughout the nebula.

While it may be true that nebular collapse was induced by the shockwave of a nearby supernova as originally suggested by Cameron and Truran (1977), and it may also be true that short-lived radioactive elements were added to the solar nebula by at least 1 nearby supernova and/or by several massive stars, these events did not necessarily occur simultaneously. The expanding matter in supernovae ejecta have a velocity dispersion that could result in some short-lived radioactive elements being deposited into the solar nebula long after the initial shockwave that induced its collapse and possibly over a timescale of many tens of thousands of years. Boss (2004) has demonstrated that the arriving nuclides will be quickly homogenized into a nebula of generally uniform composition. But what are the potential results of such a scenario for the chronology of early solar system events? At least three different scenarios appear to be possible.

The most widely accepted scenario is that the short-lived radioactive elements arrived at and were mixed into the nebula fairly early in the collapse phase. This results in our ability to use ${ }^{26} \mathrm{Al}$ as a simple chronometer, and therefore the CAIs that contain the canonical fraction of ${ }^{26} \mathrm{Al}$ really were among the first solids formed in the solar system. However, two somewhat less probable scenarios are also possible. First, if the incoming wave of radioactive elements trailed behind the initial shock wave, then ${ }^{26} \mathrm{Al}$ might have arrived in the nebula after CAI formation had already begun (Srinivasan, et al., 2000). In this scenario the ${ }^{26} \mathrm{Al}$ chronometer would be double valued: The oldest CAIs would contain no ${ }^{26} \mathrm{Al}$, but so would the youngest. Moreover, there would be a time period during which the ${ }^{26} \mathrm{Al}$ was building to the canonical value when CAIs would have increasing fractions of ${ }^{26} \mathrm{Al}$ that would mimic those produced after significant periods of decay had occurred from the canonical value. These early 
formed CAIs would be rather rare as they would have formed in the highest energy stage of the solar nebula and during the highest rate of mass accretion onto the sun, and are more likely to have been destroyed by nebular processing or by falling into the sun than later formed CAIs. The final scenario and the least likely possibility is that the ${ }^{26} \mathrm{Al}$ wave arrives just as CAIs begin to form and survive. If the wave arrived at just the correct velocity then the added ${ }^{26} \mathrm{Al}$ might just balance the decay rate of

nebular ${ }^{26} \mathrm{Al}$. In this scenario, the canonical value of ${ }^{26} \mathrm{Al}$ might have been maintained for many tens of thousands of years, thus rendering our definition of time zero an epoch rather than a distinct instant.

\section{Conclusions}

There are many uncertainties in our understanding of the dynamics and chemistry of the primitive solar nebula. The probability seems high that this turbulent, dusty environment will engender some degree of collisional, triboelectric-induced charge separation that will result in electrostatic lightning discharges. Unfortunately, quantifying such a model still relies heavily on the formulation of "reasonable" assumptions, though what is reasonable is often more a matter of opinion than fact. For this reason we have tried to state the rationale behind each of the assumptions that have gone into our lightning-induced model for the non-mass-dependent fractionation of oxygen isotopes in nebular dust as well as the range of uncertainties and possible alternatives. We have also tried to use intermediate rather than extreme values for most of the input parameters, thus rendering the model somewhat more flexible. The object of the exercise was to see if a lightning-induced model of non-mass dependent oxygen isotopic dust evolution in the primitive solar nebula is plausible. We believe that the answer to that question is an unqualified yes, the model is indeed plausible.

Is the model correct? Probably not in its present form. It is likely that several of the input parameters that we have chosen are incorrect, and might possibly be off by orders of magnitude. Do the errors in the other parameters compensate for or exacerbate one another? We do not know, though we are certain that there is sufficient flexibility in the entirety of the parameter set to accommodate significant changes in individual input variables. However, because the model is plausible, we hope to stimulate laboratory, observational and theoretical studies as discussed above related to the most uncertain of our input parameters and, even if such studies eventually prove this model to be incorrect, our knowledge of the dynamics and chemistry of the solar nebula will be greatly improved. 


\section{Acknowledgements}

The authors would like to thank the NASA Exobiology Research and Analysis Program for financial support. J.A.P. acknowledges support from the NASA Postdoctoral Program supported by funding from the Astrophysics Data Program.

\section{References}

Bell, K. R., Cassen P. M., Klahr H. H., and Henning Th. 1997. The structure and appearance of protostellar accretion disks: Limits on disk flaring. The Astrophysical. Journal 486:372-387.

Boss, A. P. 2004. Evolution of the solar nebula. VI. Mixing and transport of isotopic heterogeneity. The Astrophysical Journal, 616:1265-1277.

Cameron, A. G. W. and Truran, J. W. 1977. The supernova trigger for formation of the solar system. Icarus, 30:447-461.

Ciesla, F. J. 2007. Outward transport of high-temperature materials around the midplane of the solar nebula. Science 318:613-615.

Ciesla, F. J. 2010. The distributions and ages of refractory objects in the solar nebula. Icarus 208:455467.

Ciesla F. J. and Sandford, S. A. 2012. Organic synthesis via irradiation and warming of ice grains in the solar nebula. Science 336:452-454.

Clayton, D. 1988. Isotopic anomalies: chemical memory of galactic evolution. The Astrophysical Journal 334:191-195.

Clayton R. N. 2002. Self-shielding in the solar nebula. Nature 415: 860-861.

Clayton, R. Grossman, L. and Mayeda, T. 1973. A component of primitive nuclear composition in carbonaceous meteorites. Science 182:485-488.

Desch, S. J. and Cuzzi, J. N. 2000. The generation of lightning in the solar nebula. Icarus, 143:87-105.

Dominguez, G. 2010. A heterogeneous chemical origin for the ${ }^{16} \mathrm{O}$-enriched and ${ }^{16} \mathrm{O}$-depleted reservoirs of the early solar system. The Astrophysical Journal Letters, 713: L59-L63.

Gao, Y. Q. and Marcus, R. A. 2002. On the theory of the strange and unconventional isotopic effects in ozone formation. Journal of Chemical Physics 116: 137-154.

Gibbard, S.G., Levy, E.H., and Morfill, G.E. 1997. On the possibility of lightning in the protosolar 
nebula. Icarus, 130:517-533.

Gounelle M., Krot A. N., Nagashima K., and Kearsley A. 2009. Extreme ${ }^{16}$ O-enrichment in refractory inclusions from the Isheyevo meteorite: implication for oxygen isotope composition of the sun. Astrophysical Journal Letters, 698:L18-L22

Hartmann, L., Calvet, N., Gullbring, E., \& D'Alessio, P. 1998. Accretion and the evolution of T Tauri disks. The Astrophysical Journal, 495:385-400.

Hathorn B. C. and Marcus R. A. 1999. An intramolecular theory of the mass-independent isotope effect for ozone. I. Journal of Chemical Physics 111:4087-4100.

Hathorn B. C. and Marcus R. A. 2000. An intramolecular theory of the mass-independent isotope effect for ozone. II. Numerical implementation at low pressures using a loose transition state. Journal of Chemical Physics 113:9497-9509.

Hawley, J. F., Stone, J. M. 1998. Nonlinear evolution of the magnetorotational instability in ion-neutral disks. The Astrophysical Journal 510:758-771.

Hayatsu R. and Anders, E. 1981. Organic compounds in meteorites and their origins. Topics in Current Chemistry 99:1-37.

Heidenreich, J. E., III; Thiemens, M. H. 1985. The non-mass-dependent [NoMaD] oxygen isotope effect in the electrodissociation of carbon dioxide: a step toward understanding NoMaD chemistry. Geochimica et Cosmochimica Acta 49:1303-1306.

Hersant, F., Gautier, D., and Huré, J.-M. 2001. A two dimensional turbulent model for the solar nebula constrained by $\mathrm{D} / \mathrm{H}$ measurements in the solar system: implications for the formation of giant planets. The Astrophysical Journal 554:391-407.

Hill H. G. M. and Nuth, J. A. 2003. The Catalytic Potential of Cosmic Dust: implications for prebiotic chemistry in the solar nebula and other protoplanetary systems. Astrobiology, 3:291-304.

Itoh, S. and Yurimoto, H. 2003. Contemporaneous formation of chondrules and refractory inclusions in the early Solar System. Nature 423:728-731.

Kimura, Y., Nuth, J.A., Chakraborty, S., and Thiemens, M.H. 2007. Non-mass-dependent oxygen isotopic fractionation in smokes produced in an electrical discharge. Meteoritics and Planetary Science 42:1429-1439.

Kobayashi S., Imai H., and Yurimoto H. 2003. New extreme ${ }^{16}$ O-rich reservoir in the early solar system. Geochemical Journal 37:663-669

Krankowsky, K. and Mauersberger, K. 1996. Heavy ozone-a difficult puzzle to solve. Science 
274: $1324-1325$.

Krot, A.N., Nagashima, K, Ciesla, F., Meyer, B. S., Hutcheon, I. D., Davis, A. M. Huss, G. R. and Scott, E. R. D. 2010. Oxygen isotopic composition of the Sun and mean oxygen isotopic composition of the protosolar silicate dust: Evidence from refractory inclusions. The Astrophysical Journal 713:1159-1166.

Lide, D. R. 1996. CRC handbook of chemistry and physics, 77th edition. Boca Raton, FL: CRC Press. pp. 14-33.

Llorca, J. and Casanova, I. 2000. Formation of carbides and hydrocarbons in chondritic interplanetary dust particles: A laboratory study. Meteoritics and Planetary Science 33:243-251.

Love, S.G., Keil, K., and Scott, E.R.D. 1995. Electrical discharge heating of chondrules in the solar Nebula. Icarus 115:97-108.

Lyons J. R. and Young E. D. 2005. CO self-shielding as the origin of oxygen isotope anomalies in the early solar nebula. Nature 435: 317-320.

Marcus, R. A. 2004. Mass independent isotope effect in the earliest processed solids in the solar system: A possible chemical mechanism. Journal of Chemical Physics 121:8201-8211.

Mauersberger, K., Erbacher, B., Krankowsky, D., Günther, J. and Nickel, R. 1999. Ozone isotope enrichment: isotopomer-specific rate coefficients. Science 283: 370-372.

McKeegan, K. D., Kallio, A. P. A., Heber, V. S., Jarzebinski, G., Mao, P. H., Coath, C. D. Kunihiro, T., Weins, R. C., Nordholt, J. E., Moses, R. W., Reisenfeld, D. B., Jurewicz, A. J.G., and Burnett, D. S. 2011. The oxygen isotopic composition of the sun inferred from captured solar wind. Science 332:1528-1532.

Nuth, J. A. 2009. A simple mechanism for fractionating oxygen isotopes in the solar system (abstract \# 5086). Annual Meeting of the Meteoritical Society, Nancy France, July 2009.

Nuth, J. A. Rietmeijer, F. J. M. and Hill, H. G. M. 2002. Condensation processes in astrophysical environments: the composition and structure of cometary grains. Meteoritics and Planetary Science 37:1579-1590.

Nuth, J. A., Hallenbeck, S. L. and Rietmeijer, F. J. M. 1998. Interstellar and interplanetary grains: recent developments and new opportunities for experimental chemistry._Earth, Moon and Planets 80:73-112.

Nuth, J. A. and Hill, H. G. M. 2004. Planetary accretion, oxygen isotopes and the central limit theorem. Meteoritics \& Planetary Science 39:1957-1965. 
Nuth, J. A., Johnson, N. M. and Manning, S. 2008a. A self-perpetuating catalyst for the production of complex organic molecules in protostellar nebulae. In Organic Matter in Space, Proceedings IAU Symposium No. 251, 2008 edited by S. Kwok \& S. Sandford. pp. 403-408.

Nuth, J. A., Johnson, N. M. and Manning, S. 2008b. A self-perpetuating catalyst for the production of complex organic molecules in protostellar nebulae. The Astrophysical Journal Letters 673: L225-L228

Nuth, J. A., Kimura, Y. Lucas, C., Ferguson, F. T. and Johnson, N. M., 2010. The formation of graphite whiskers in the primitive solar nebula. The Astrophysical Journal Letters. 710:L98-L101.

Paquette, J. A. and Nuth, J. A. 2012. A model of oxygen isotopic fractionation in the proto-solar nebula due to nebular lightning (abstract\#5079). 79th Annual Meeting of the Meteoritical Society.

Shi X., Yin Q.-Z., Wiens R., Ng C.-Y. 2012. Isotope composition of atomic oxygen and branching ratio from $\mathrm{CO}$ predissociation: implications for oxygen isotope evolution in the early solar nebula (abstract\#1403). Lunar and Planetary Science Conference.

Simon, J. I, Hutcheon, I. D., Simon, S. B., Matzel, J. E. P., Ramon, E. C., Weber, P. K., Grossman, L. and DePaolo, D. J. 2011. Oxygen isotope variations at the margin of a CAI records circulation within the solar nebula. Science 331:1175-1178.

Srinivasan G., Papanastassiou D. A., Wasserburg G. J., Bhandari N., and Goswami J. N. 2000. Reexamination of ${ }^{26} \mathrm{Al}^{26}{ }^{26} \mathrm{Mg}$ systematics in the Piplia Kalan eucrite (abstract \#1795). 31st Lunar and Planetary Science Conference. CD-ROM.

Thiemens M. H. and Jackson T. 1987. Production of isotopically heavy ozone by ultraviolet light photolysis of oxygen. Geophysical Research Letters 6:624-627.

Thiemens M. H. 1996. Mass-independent isotope effects in chondrites; The role of chemical processes. In Chondrules and the Protoplanetary Disk, edited by Hewins R. H., Jones R. H., and Scott E. R. D. (Boston: Cambridge University Press). pp. 107-118.

Thiemens M. H. and Heidenreich J. E. 1983. The mass-independent fractionation of oxygen: A novel isotope effect and its possible cosmochemical implications. Science 219:1073-1075.

Wiens, R.C., Huss, G. R. and Burnett, D.S. 1999. The solar oxygen-isotopic composition: Predictions and implications for solar nebula processes. Meteoritics \& Planetary Science 34: 99-107.

Young, E. D. 2007. Time-dependent oxygen isotope effects of CO self shielding across the protoplanetary disk. Earth and Planetary Science Letters 262:468-483. 
Yurimoto, H. and Kuramoto, K. 2004. Molecular cloud origin for the oxygen isotope heterogeneity in the solar system. Science 305:1763-1766.

\section{Figure Captions}

Figure 1 is a typical three-isotope plot of oxygen isotopes in CAIs and chondrules, and in various meteorite types (note that the label "O chondrules" refers to chondrules from ordinary chondrites, "E chondrules" from enstatite chondrites, "C chondrules" from carbonaceous chondrites and TF is the Terrestrial Fractionation line). The CAI line delineates the evolution of oxygen in solids either by evaporation + exchange + recondensation or by photodissociation. Any mineral phase on the CAI line can react and fractionate along slope 0.52 lines to broaden the CAI line. Finally, if the oxygen isotopic composition of the gas and dust were originally the same, then cometary water (representing the nebular gas) would evolve to more negative values as processing proceeded to increase the dust in ${ }^{17} \mathrm{O}$ and ${ }^{18} \mathrm{O}$. However, since there is 20-30 times more gas than dust, (Depending on the amount of dust that has settled to the nebular midplane) the affect on the isotopic composition of the gas is much less dramatic than on the composition of the solids. This figure is modified from Nuth et al. (1998).

Figure 2 Each lightning bolt is assumed to be a cylinder $100 \mathrm{~km}$ long by $7 \mathrm{~m}$ across. If we were to change the dimensions of the standard lightning bolt it would mean that more (less) energy would be dissipated for a longer (or shorter) bolt, thus decreasing (increasing) the number of bolts per second. However, the change in the dimensions of the bolt would not change the overall nebular volume processed through the total number of lightning strokes in the nebula at a given time since more, smaller (or fewer, larger) bolts would affect the same volume based solely on the quantity of energy dissipated as lightning per unit time.

Figure 3 shows the change in the average oxygen isotopic composition of dust in the primitive solar nebula as a function of time, relative to an arbitrary initial isotopic composition of oxygen in the dust based on the assumptions stated on the plot. Note that the $\delta^{17} \mathrm{O}$ of the dust changes very rapidly and reaches 10 to 30 per mil above the initial oxygen isotopic composition within the first few hundred thousand years. This model demonstrates that nebular dust could reach about 50 per mil above solar at a million years after the process begins but does not exceed 70 per mil above the initial value even at 10 
million years. The rapid drop in the rate of change in the oxygen isotopic composition of the dust is due to the rapid drop in accretion powered lightning.

Figure 4. Plotted here are the mean, the mean plus or minus one standard deviation, the minimum and maximum for the number of times that dust in 10,000 distinct, closed volumes was struck by lightning as a function of time for the lightning frequency given in Figure 2. For each volume at each time step the probability of a lightning strike was computed stochastically based on the lightning frequency shown in Figure 2. Unlike the results presented in Figure 3, we assumed that there was no mixing between volumes: This assumption represents the other extreme as compared to perfect mixing and demonstrates that under these circumstances there can be as much as a factor of two difference in the oxygen isotopic composition of co-existing nebular volumes.

Figure 5. The number of lightning hits on each of the 1000 volumes followed during a single Monte Carlo run at times ranging from 10 through 5,000,000 years. The first non-zero hit bin does not occur until the model reaches 100 years in this particular run. Even at 10,000 years, most bins have only been struck once or twice, $\sim 20 \%$ are still unstruck, $\sim 20 \%$ were struck 5 times, five bins were struck 7 times and 1 was struck 8 times (the maximum). At 100,000 years the system has almost reached a normal distribution with the average volume struck nearly 100 times, but with some struck 33 times while one was struck only 6 times. At 1 and 5 million years, the distributions are quite symmetric with about 95 and 120 average hits, and a full width from 68 to 129 and 98 to 157 hits, respectively.

Figure 6. This illustrates the average result when 1000 separate volumes were run through the model in reverse. That is, we calculated the probability of a lightning strike based on the lightning frequency at 5 million years initially and increased the probability as the calculations progressed. We continued the calculation until we reached the maximum lightning frequency at time zero. This demonstrates that it is possible for a packet of material to fall into the solar nebula quite late in its evolution and still have some fraction of the original material survive unscathed under the assumptions of the current model. 
Figure 1

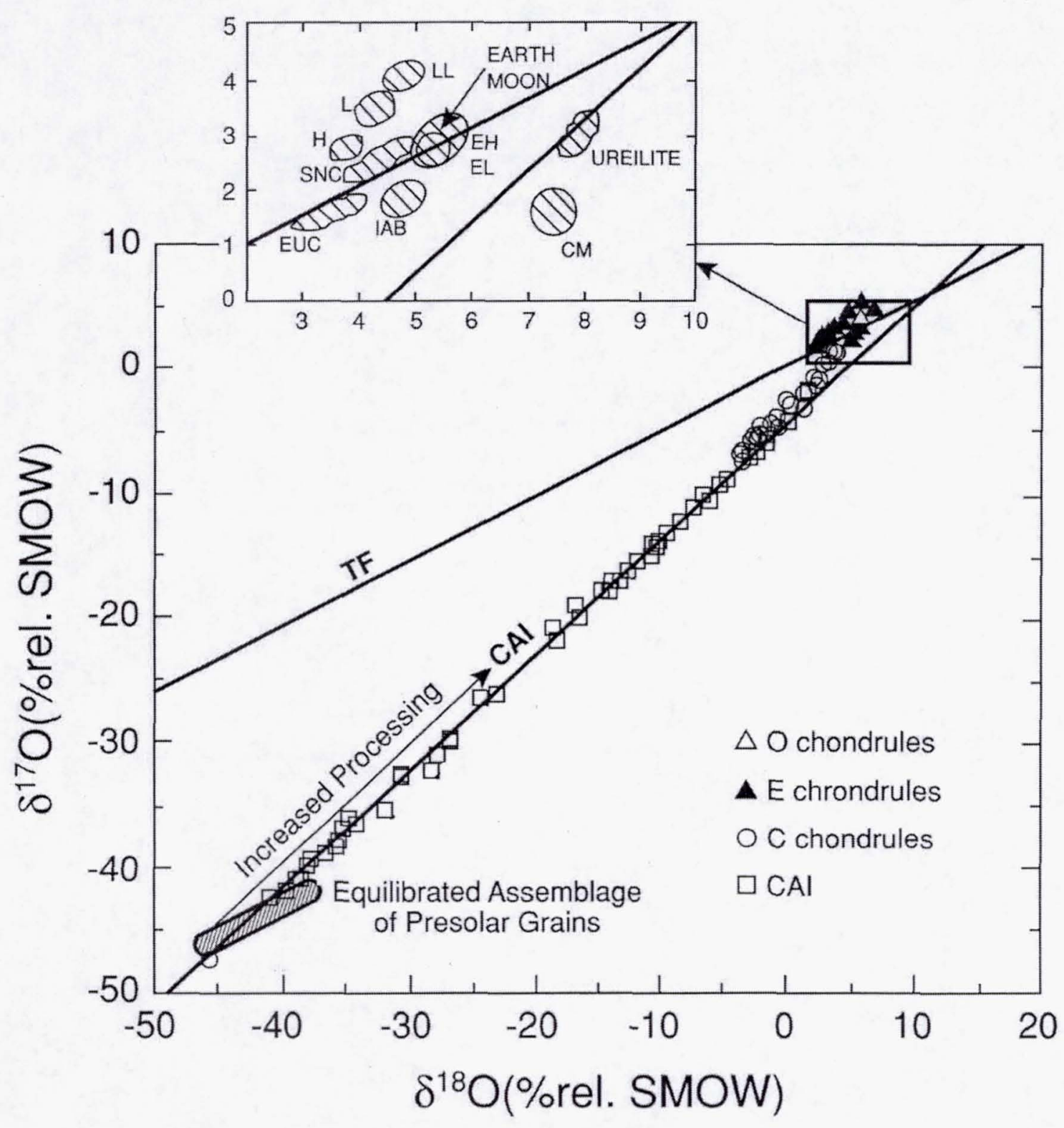




\section{Figure 2}

Number of Bolts per Second Throughout the Inner Nebula vs. Time

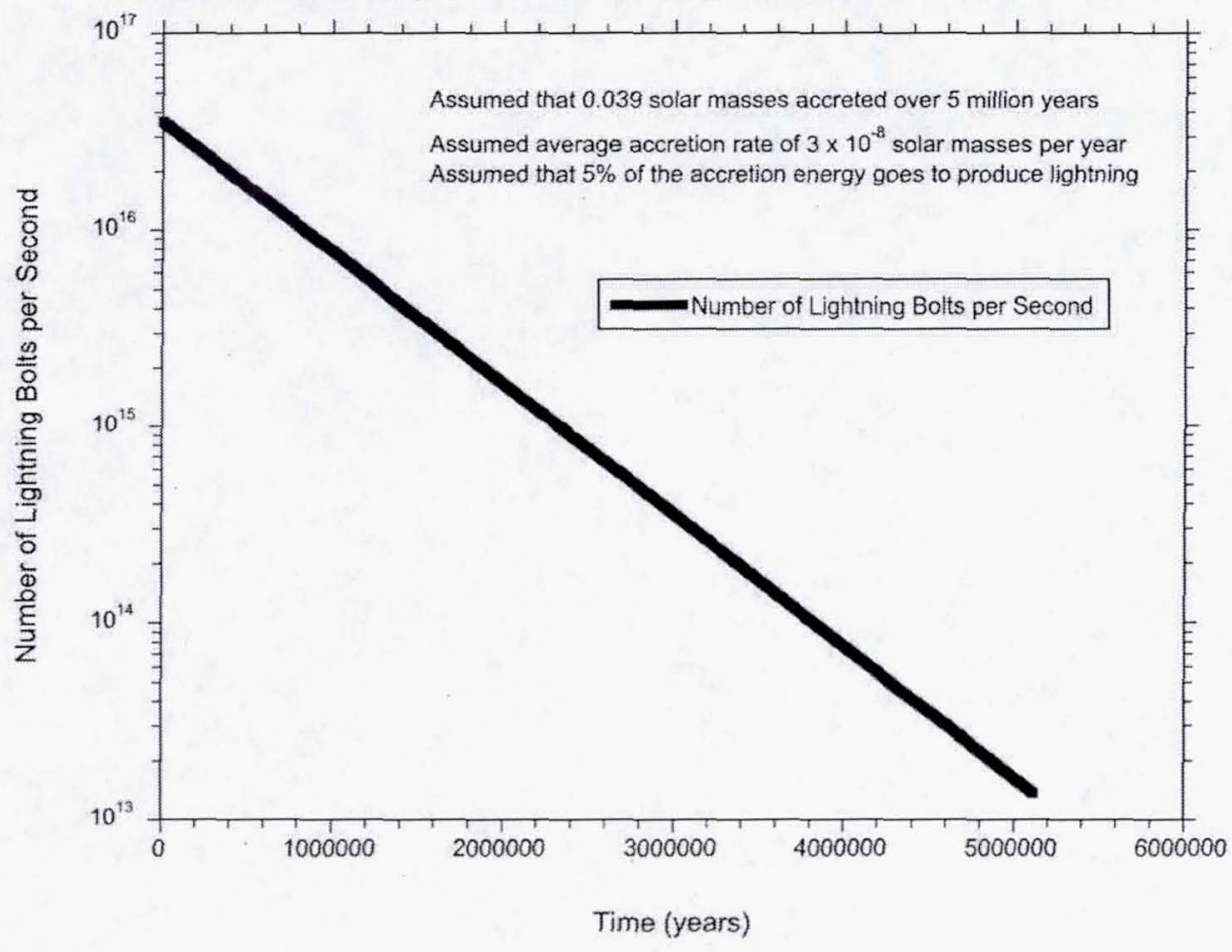




\section{Figure 3}

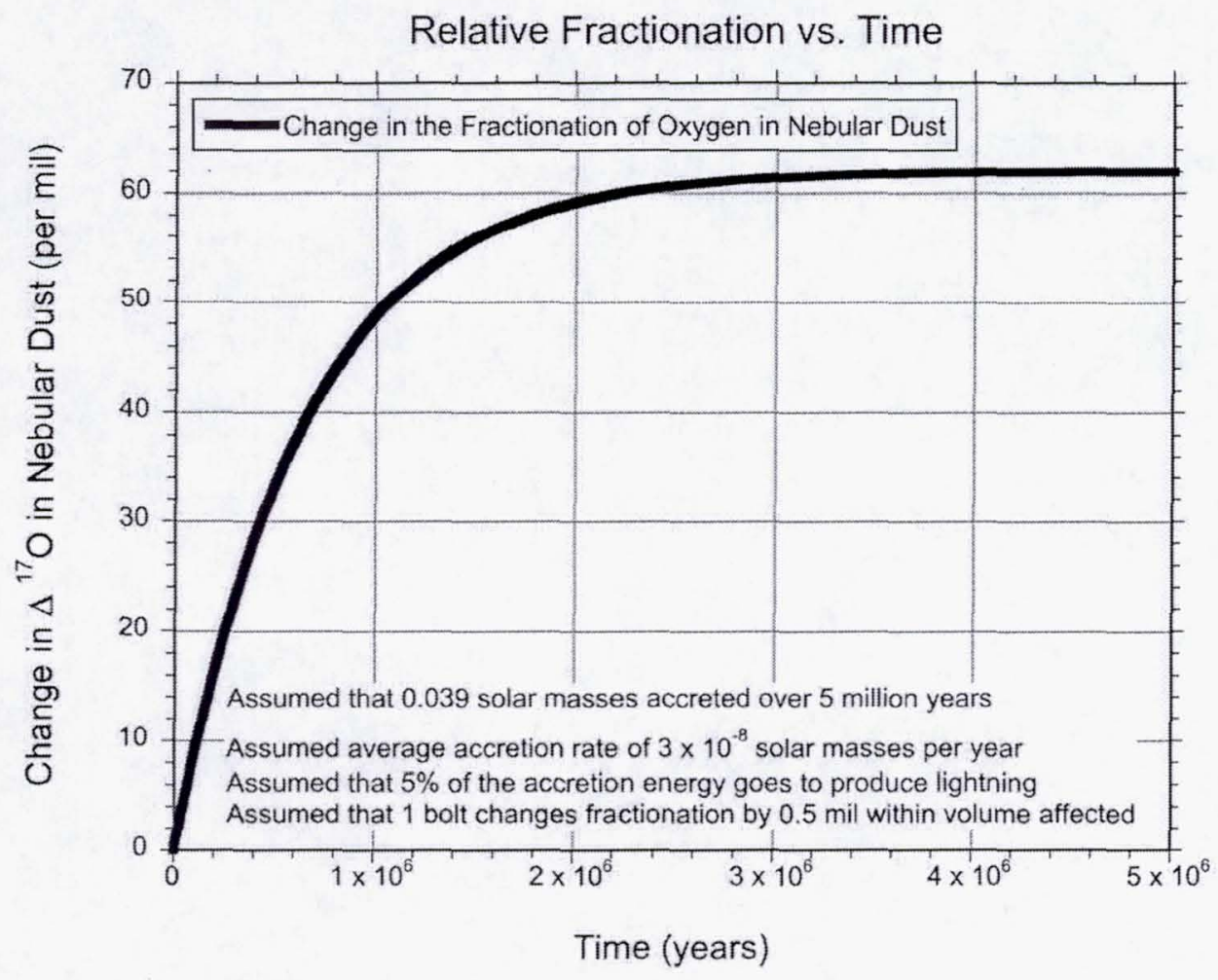


Figure 4

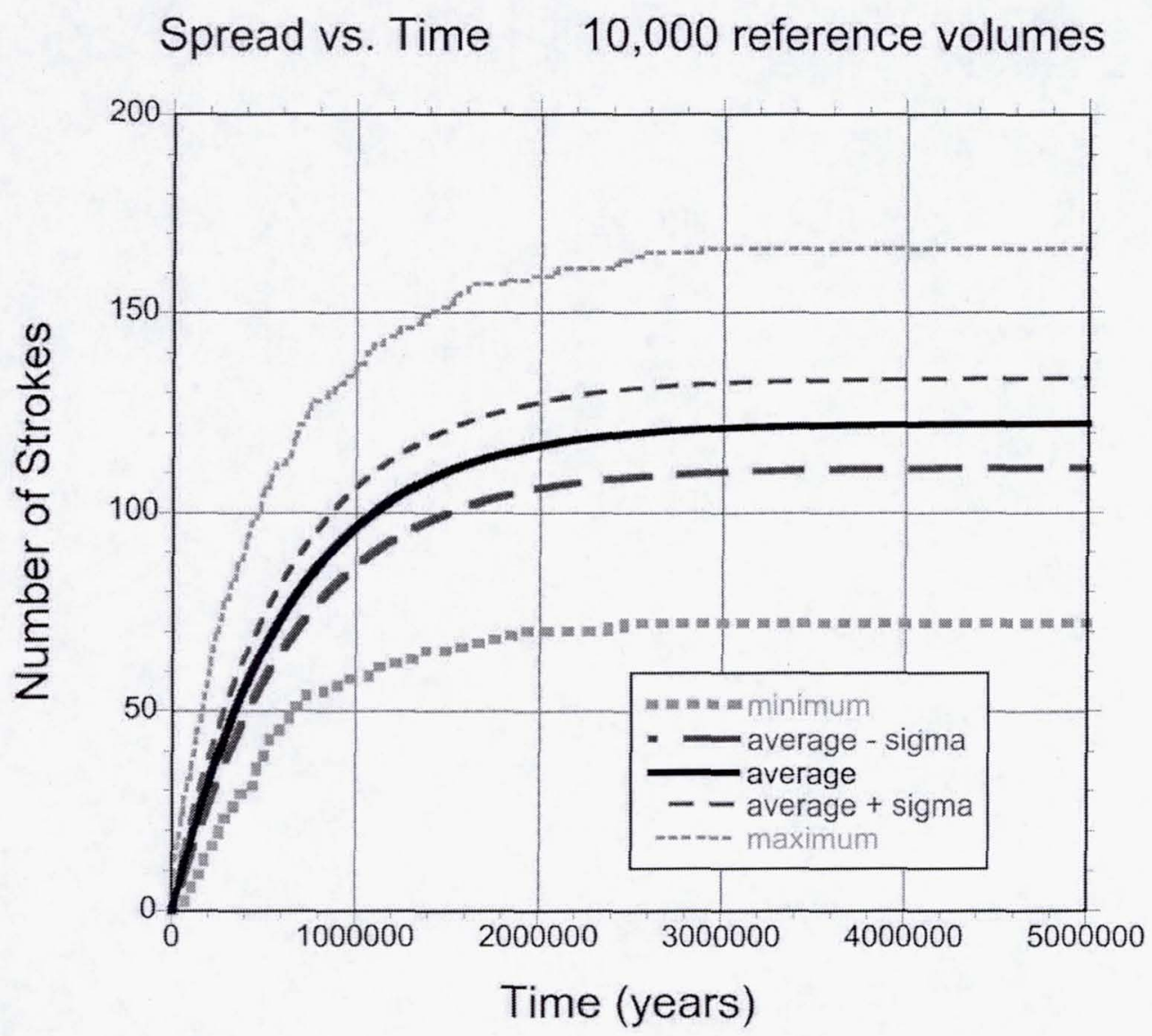


Figure 5

Distributions at Selected Times
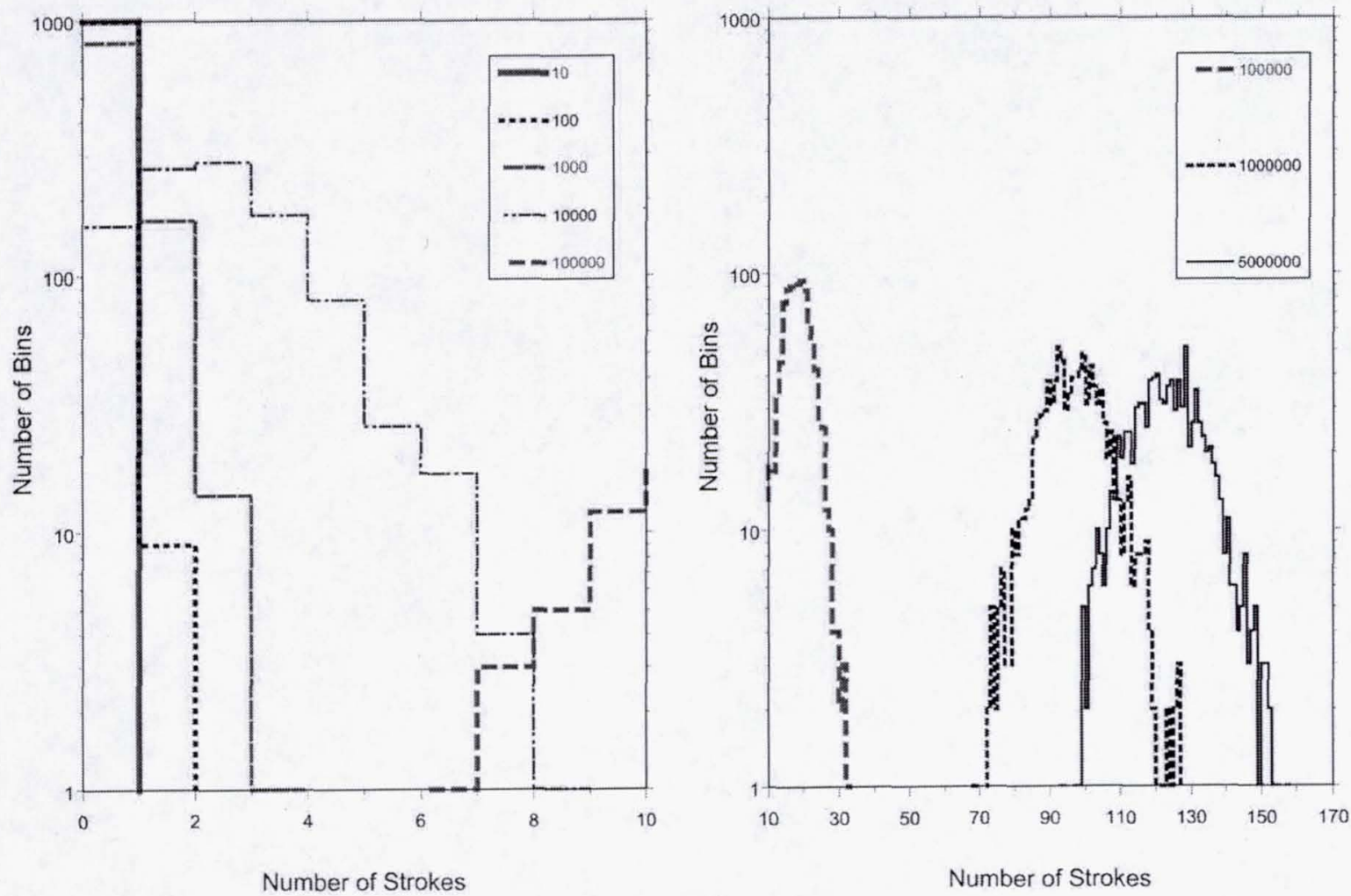
Figure 6

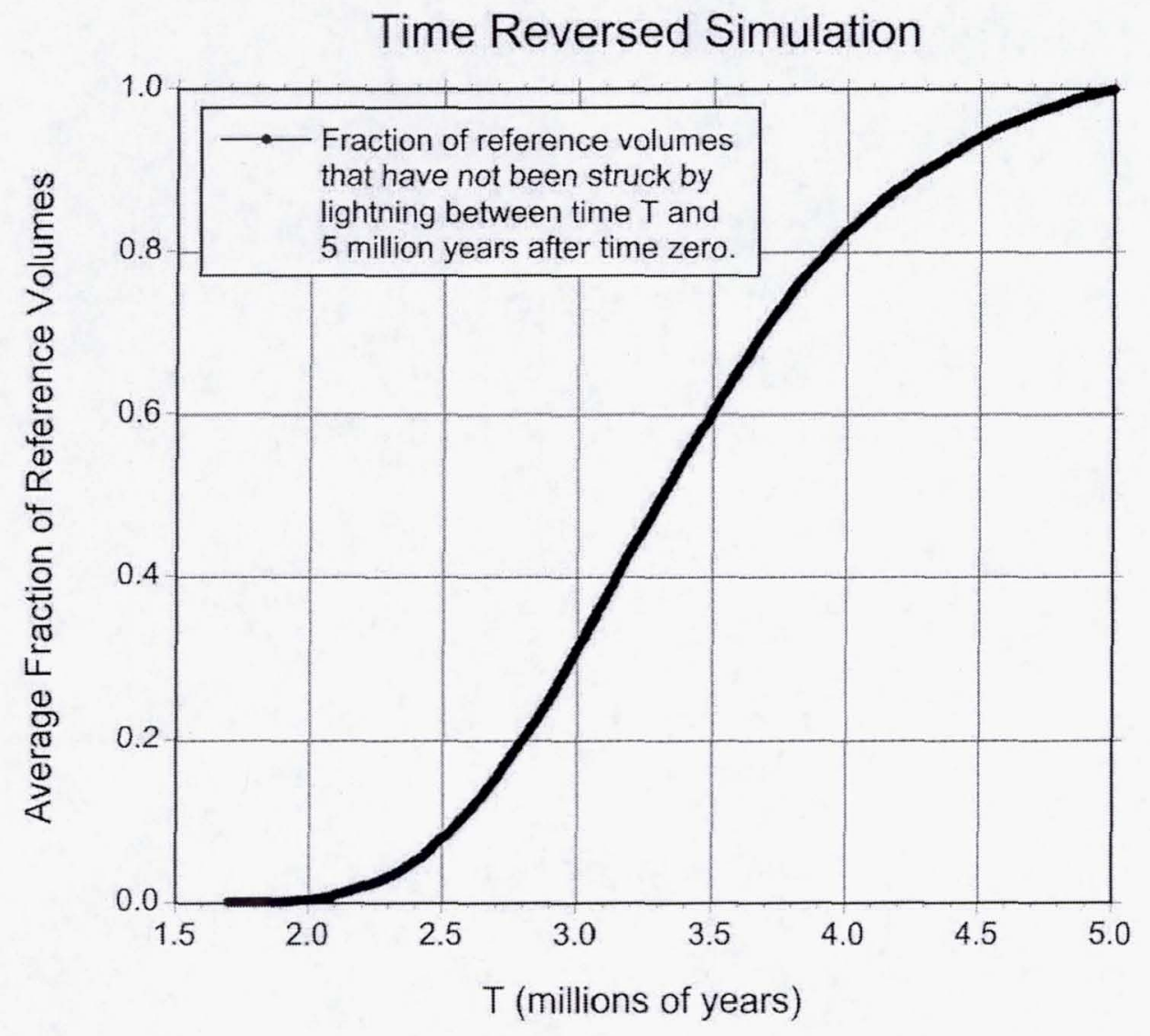

